\title{
Heat and mass transfer in evaporating turbulent drop-laden flow
}

\author{
R. Groll \\ Center of Applied Space Technology and Microgravity, \\ University of Bremen, Germany
}

\begin{abstract}
This work deals with the computational modelling of the mass transition of evaporating liquid drop-laden gas flows. In the present study the evaporation model due to Abramzon and Sirignano (1989) has been extended by introducing an additional transport equation for a newly defined quantity $\bar{a}$, defined as the phase-interface surface fraction. This allows the change in the drop diameter to be quantified in terms of a probability density function. The source term in the equation describing the dynamics of the volumetric fraction of the dispersed phase $\alpha^{D}$ is related to the evaporation time scale $\tau_{\Gamma}$.

Keywords: Euler/Euler approach, Euler/Lagrange approach, gas/liquid flow, evaporation model, heat and mass transfer, volume and surface fraction.
\end{abstract}

\section{Introduction}

The dimensionless numbers characterizing the heat and mass exchange process are Nusselt number $N u$ and Sherwood number $S h$. Several models (e.g. Renksizbulut and Yuen [1] and Park et al. [2]) are based on the correlation model of Ranz and Marshall [3] and have been developed to model the parameters. The evaporation rate model of Abramzon and Sirignano [4] considers additionally the latent heat flux of the evaporated liquid leaving the droplet. The correct capturing of the gas phase humidity requires the liquid vapor mass ratio $Y$, influenced by convective, conductive, turbulent and thermal diffusive effects, to be computed from an appropriate transport equation in addition to the equation governing the temperature field $T$.

In an evaporating process the mass transfer rate on the drop surface depends on the drop size. The polydispersed spray consists of drops with different diameters. 


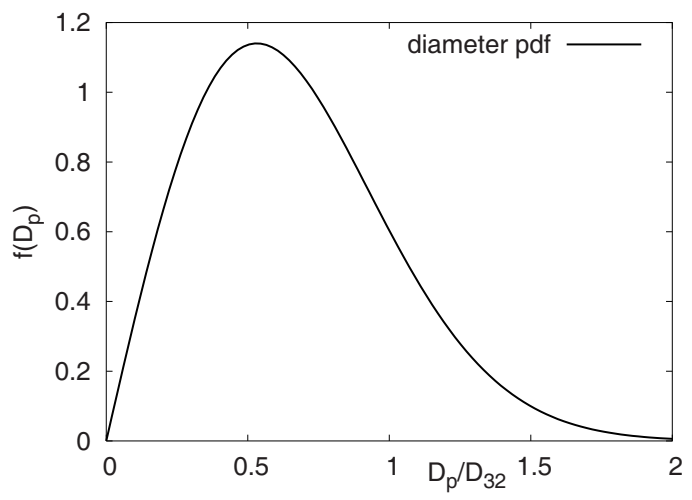

Figure 1: PDF of a normalized particle drop diameter in a polydispersed spray.

To determine the mass transfer, i.e. the evaporation rate for such a case, the drop diameter distribution of the spray is necessary. Using the following approach based on a particle diameter probability density function (PDF) (see Fig. 1 for its graphical representation):

$$
f\left(\frac{D_{p}}{\mathrm{E}\left(D_{p}\right)}\right)=\frac{\pi}{2} \frac{D_{p}}{\mathrm{E}\left(D_{p}\right)} e^{-\frac{\pi}{4}} \frac{D_{p}^{2}}{\mathrm{E}\left(D_{p}\right)^{2}} \quad \text { with } f\left(D_{p}\right)=\frac{9}{8} \pi \frac{D_{p}}{D_{32}^{2}} e^{-\frac{9}{16} \pi \frac{D_{p}^{2}}{D_{32}^{2}}}
$$

depending on the Sauter mean diameter,

$$
D_{32}=\frac{\mathrm{E}\left(D_{p}^{3}\right)}{\mathrm{E}\left(D_{p}^{2}\right)}=\frac{6 \bar{\alpha}^{D}}{\bar{a}} \Leftrightarrow \frac{\bar{a}}{\pi \mathrm{E}\left(D_{p}^{2}\right)}=\frac{\bar{\alpha}}{\frac{\pi}{6} \mathrm{E}\left(D_{p}^{3}\right)}
$$

with the volumetric fraction $\alpha$ and the newly defined variable $\bar{a}$ being the phaseinterface surface fraction,

$$
\alpha=\frac{V_{\text {liquid }}}{\left(V_{\text {liquid }}+V_{\text {gas }}\right)} \quad a=\frac{A_{\text {phase interface }}}{\left(V_{\text {liquid }}+V_{\text {gas }}\right)}
$$

the mass transfer rate are calculated as shown in the further work. The expectation values of the squared and cubic diameters in terms of the Sauter mean diameter only, result from this modelled PDF (eq. 1), e.g. Groll et al. [5]:

$$
\mathrm{E}\left(D_{p}\right)=\frac{2}{3} D_{32} \quad \mathrm{E}\left(D_{p}^{2}\right)=\frac{16}{9 \pi} D_{32}^{2} \quad \mathrm{E}\left(D_{p}^{3}\right)=\frac{16}{9 \pi} D_{32}^{3}
$$

A time dependent relation has to be defined to calculate the change of the drop diameter when simulating motion of a polydispersed phase. The change of the expectation value of the diameter squared is presumed to be constant in accordance 
to the $d^{2}$-law (Kastner [6]):

$$
\frac{\mathrm{d}}{\mathrm{d} t} \mathrm{E}\left(D_{p}^{2}\right)=-\Gamma
$$

Integration of this equation results in the time-dependent solution for the drop diameter:

$$
D_{p}(t)=\sqrt{D_{p}^{2}(0)-\Gamma t} .
$$

Substitution of the expectation value with the given density function definition

$$
-\Gamma=\frac{\mathrm{d}}{\mathrm{d} t} \mathrm{E}\left(D_{p}^{2}\right)=\frac{16}{9 \pi} \frac{\mathrm{d}}{\mathrm{d} t} D_{32}^{2}=\frac{32}{9 \pi} \dot{D}_{32} \frac{\mathrm{d}}{\mathrm{d} t} D_{32} \Rightarrow \dot{D}_{32}=-\frac{9 \pi}{32} \frac{\Gamma}{D_{32}}
$$

reveals that the deviation of the Sauter mean diameter depends on the evaporation constant of the $d^{2}$-law. Based on this formulation, the following equation has been derived:

$$
\frac{\mathrm{d}}{\mathrm{d} t} \mathrm{E}\left(D_{p}^{3}\right)=\frac{16}{9 \pi} \frac{\mathrm{d}}{\mathrm{d} t} D_{32}^{3}=\frac{16}{9 \pi}\left(\dot{D}_{32} D_{32}^{2}+D_{32} \frac{\mathrm{d}}{\mathrm{d} t} D_{32}^{2}\right)=-\frac{3}{2} \Gamma D_{32} .
$$

With this expression, the time-dependent change of an expected drop volume is defined. The outcome of the last equation is used for the determination of the mass transfer of a drop with the expected mass $\bar{m}_{p}$ :

$$
\frac{\mathrm{d} \bar{m}_{p}}{\mathrm{~d} t}=\frac{\pi}{6} \rho^{D} \frac{\mathrm{d}}{\mathrm{d} t} \mathrm{E}\left(D_{p}^{3}\right)=-\frac{\pi}{4} \rho^{D} D_{32} \Gamma .
$$

Based on the evaporation model due to Abramzon and Sirignano [4], the mass transfer rate at the surface of a drops cloud representing a function of the modified Sherwood number $S h^{*}$ and the mass transfer coefficient $B_{M}$ is to be determined by using the following equations:

$$
\dot{m}=\pi D_{21} \rho^{C} D_{\alpha \beta} \mathrm{Sh}^{*} \ln \left(1+B_{M}\right)
$$

The definitions of the Sherwood number and its modification and the mass transfer coefficient are given in Section 3. By equalizing the PDF-dependent mass transfer rate and the modelled mass transfer rate, the evaporation factor $\mathrm{Sh}^{*} \ln \left(1+B_{M}\right)$ in the model of Abramzon and Sirignano [4] is defined as:

$$
\begin{gathered}
\frac{\pi}{4} \rho^{D} D_{32} \Gamma=-\dot{m}_{p}=\dot{m}=\pi D_{21} \rho^{C} D_{\alpha \beta} \operatorname{Sh}^{*} \ln \left(1+B_{M}\right) \\
\Rightarrow \Gamma=4 \frac{D_{21}}{D_{32}} \frac{\rho^{C}}{\rho^{D}} D_{\alpha \beta} \operatorname{Sh}^{*} \ln \left(1+B_{M}\right) .
\end{gathered}
$$

In accordance to the $d^{2}$-law, the evaporation constant should be independent of the particle diameters. This fact brings an additional constraint to the expectation values of the probability function: $D_{21} / D_{32}=$ const. The given PDF fulfills this 
condition as follows:

$$
\frac{D_{21}}{D_{32}}=\frac{\mathrm{E}\left(D^{2}\right) \mathrm{E}\left(D^{2}\right)}{\mathrm{E}(D) \mathrm{E}\left(D^{3}\right)}=\frac{8}{3 \pi} .
$$

With the proposal of Abramzon and Sirignano, the time-dependent modelling of the drop diameter probability function is closed. Combining this information with the relation of the change of the specific volume $\alpha$ and the specific surface $a$, described in section 2 , the transport equations are developed.

\section{Evaporation progress}

The model developed serves for calculation of the evaporation rate of spherical water drops. Water is a liquid dispersed phase, which satisfies the $d^{2}$-law (e.g. Kastner [6]). Keeping in mind the definition of the life time of a drop $T$ and its diameter loss rate, it is known, that each drop with a diameter

$$
D_{p}<(\Gamma T)^{\frac{1}{2}}
$$

is evaporated completely. To determine the number of the evaporated drops in a cloud, the probability density function has to be integrated in the following way:

$$
\int_{0}^{(\Gamma T)^{\frac{1}{2}}} f\left(D_{p}\right) \mathrm{d} D_{p} .
$$

Consequently, the time change of the particle number is obtained as follows:

$$
\begin{aligned}
\frac{\mathrm{d} \bar{n}}{\mathrm{~d} t} & =-\lim _{T \rightarrow 0}\left[\frac{\bar{n}}{T} \int_{0}^{(\Gamma T)^{\frac{1}{2}}} f\left(D_{p}\right) \mathrm{d} D_{p}\right]=\lim _{T \rightarrow 0}\left[\frac{\bar{n}}{T}\left(e^{-\frac{9 \pi}{16} \frac{\Gamma T}{D_{32}^{2}}}-1\right)\right] \\
& =\lim _{T \rightarrow 0}\left[\frac{\bar{n}}{T}\left(e^{m T}-1\right)\right] \quad ; \quad m=-\frac{9 \pi}{16} \frac{\Gamma}{D_{32}^{2}} \\
& =\lim _{T \rightarrow 0}\left[\frac{\bar{n}}{T}\left((1+m T)^{\frac{1}{m T}} \cdot m T-1\right)\right] \\
& =\lim _{T \rightarrow 0}\left[\frac{\bar{n}}{T} m T\right]=\bar{n} m=-\frac{9 \pi}{16} \frac{\Gamma}{D_{32}^{2}} \bar{n} .
\end{aligned}
$$

The time change of the volumetric fraction $\bar{\alpha}^{D}$ is calculated by using the results represented by Eqs. (8) and (15):

$$
\begin{aligned}
\frac{\mathrm{d} \bar{\alpha}^{D}}{\mathrm{~d} t} & =\frac{\pi}{6} \mathrm{E}\left(D_{p}^{3}\right) \frac{\mathrm{d}}{\mathrm{d} t} \bar{n}+\bar{n} \frac{\pi}{6} \frac{\mathrm{d}}{\mathrm{d} t} \mathrm{E}\left(D_{p}^{3}\right) \\
& =-\frac{\pi}{6} \frac{16}{9 \pi} D_{32}^{3} \cdot \frac{9 \pi}{16} \frac{\Gamma}{D_{32}^{2}} \bar{n}-\bar{n} \frac{\pi}{6} \cdot \frac{3}{2} \Gamma D_{32}=-\frac{45}{32} \pi \frac{\Gamma \bar{\alpha}^{D}}{D_{32}^{2}}
\end{aligned}
$$


The time change of the Sauter mean diameter (Eq. 2) consists of the deviations of the volumetric fraction and the surface fraction:

$$
\dot{D}_{32}=\frac{6}{\bar{a}^{2}}\left(\bar{a} \frac{\mathrm{d} \bar{\alpha}^{D}}{\mathrm{~d} t}-\bar{\alpha}^{D} \frac{\mathrm{d} \bar{a}}{\mathrm{~d} t}\right)=D_{32}\left(\frac{1}{\bar{\alpha}^{D}} \frac{\mathrm{d} \bar{\alpha}^{D}}{\mathrm{~d} t}-\frac{1}{\bar{a}} \frac{\mathrm{d} \bar{a}}{\mathrm{~d} t}\right)
$$

Utilizing the results following from the Eqs. (7) and (17), the change of the surface fraction can also be formulated in terms of the evaporation constant and the Sauter mean diameter:

$$
\frac{1}{\bar{a}} \frac{\mathrm{d} \bar{a}}{\mathrm{~d} t}=\frac{1}{\bar{\alpha}^{D}} \frac{\mathrm{d} \bar{\alpha}^{D}}{\mathrm{~d} t}-\frac{1}{D_{32}} \frac{\mathrm{d} D_{32}}{\mathrm{~d} t}=-\frac{9 \pi}{8} \frac{\Gamma}{D_{32}^{2}} \Rightarrow \frac{\mathrm{d} \bar{a}}{\mathrm{~d} t}=-\frac{9 \pi}{8} \frac{\Gamma}{D_{32}^{2}} \bar{a} .
$$

The source terms of the $\alpha$-equation (Eq. 17) and the $a$-equation (Eq. 19) stay in following relationship:

$$
\frac{1}{\bar{\alpha}^{D}} \frac{\mathrm{d} \bar{\alpha}^{D}}{\mathrm{~d} t}=\frac{5}{4} \cdot \frac{1}{\bar{a}} \frac{\mathrm{d} \bar{a}}{\mathrm{~d} t} .
$$

Obviously, the source terms in both transport equations can be formulated in terms of the same parameter: $\tau_{\Gamma}$ (Eq. 23). With the definition of the particle diameter probability density function as the starting point, the evaporation process is finally modelled by the following two transport equations:

$$
\begin{aligned}
\partial_{t}\left(\rho^{D} \bar{\alpha}^{D}\right)+\partial_{j}\left(\rho^{D} \bar{\alpha}^{D}<u_{j}^{D}>^{D}\right) & =-\frac{5}{4} \rho^{D} \tau_{\Gamma}^{-1} \bar{\alpha}^{D} \\
\partial_{t}\left(\rho^{D} \bar{a}\right)+\partial_{j}\left(\rho^{D} \bar{a}<u_{j}^{D}>^{D}\right) & =-\rho^{D} \tau_{\Gamma}^{-1} \bar{a}
\end{aligned}
$$

The convective transport in both equations is defined by the volume-fractionweighted averaged particle velocity $\left\langle u_{i}^{D}\right\rangle^{D}$ (e.g. Politis [7]). The first equation (Eq. 21) originates from the well-known mass balance of the dispersed phase with a mass transfer defining source term. The second equation (Eq. 22) governs the surface fraction of the dispersed phase, being the synonym for the cloud surface per volume. The evaporation time scale $\tau_{\Gamma}$, the source terms of this evaporationdescribing two-equation model depend upon, reads:

$$
\tau_{\Gamma}=-\bar{a}\left(\frac{\mathrm{d} \bar{a}}{\mathrm{~d} t}\right)^{-1}=\frac{8}{9 \pi} \frac{D_{32}^{2}}{\Gamma}
$$

Accordingly, the transport of both quantities $\alpha^{D}$ and $a$ depends on the evaporation constant $\Gamma$, which is given by the $d^{2}$-law. Introducing the definition of the evaporation constant, the final expression serving for the determination of the evaporation time scale is given by:

$$
\tau_{\Gamma}=\frac{D_{32}^{2}}{12} \frac{\rho^{D}}{\rho^{C}}\left[D_{\alpha \beta} \operatorname{Sh}^{*} \ln \left(1+B_{M}\right)\right]^{-1} .
$$

By solving the equations of the $\alpha$-a-model the evaporation rate of a spray stream can be quantified. In such a way, the mass balance of a two-phase flow is completely satisfied. 


\section{Radiation, heat and mass transfer}

In accordance to the transport of the absolute humidity $Y$ the density of the continuous phase consisting of the vapor and the carrier gas phase (p.e. air) changes depending on that value:

$$
\rho^{C}=\frac{\rho^{\text {vapor }}}{Y}=\frac{\rho^{\text {air }}}{1-Y} .
$$

The radiative heat flux per volume $\dot{q}_{\mathrm{rad}}^{D}$, absorbed by a particle, is defined in terms of its surface area per volume $a$, the absorption coefficient of the dispersed phase $\epsilon_{\mathrm{abs}}^{D}$ and the difference of the fourth powers of the absolute temperatures.

$$
\dot{q}_{\mathrm{rad}}^{D}=a \epsilon_{\mathrm{abs}}^{D} \sigma\left[\left(T^{C}\right)^{4}-\left(T^{D}\right)^{4}\right], \quad \epsilon_{\mathrm{abs}}^{D}=\epsilon_{\mathrm{abs}}^{\mathrm{water}}=0,92
$$

with the Stephan-Boltzmann constant $\sigma=5,67051 \cdot 10^{-8} \mathrm{~W} /\left(\mathrm{m}^{2} \mathrm{~K}\right)$. This term represents an additional source term in the equation of thermal transport. Due to the equivalence of emission and absorption coefficients, the radiative flux can take negative values, if the dispersed phase is on the higher temperature level compared to the continuous one.

As defined in the evaporation model of Abramzon and Sirignano [4], the heat transfer at the surface of an evaporating drop depends on the latent heat $L\left(T^{D}\right)$, the temperature difference and the mass transfer $\dot{m}$

$$
Q_{L}=\dot{m}\left[\frac{c_{p}^{D}\left(T^{C}-T^{D}\right)}{B_{T}}-L\left(T^{D}\right)\right] .
$$

The heat transfer coefficient $B_{T}$ is to be computed from the "Abramzon and Sirignano"-model algorithm. By utilizing Eq. (21), this heat source term can be expressed in terms of $\tau_{\Gamma}$ in a volume specific way.

Using the following definition of the evaporation enthalpy

$$
\Delta h_{v}=\frac{R_{0}}{M^{\text {vapor }}}\left(\frac{1}{T^{D}}-\frac{1}{T_{\text {ref }}}\right)^{-1} \ln \frac{p_{\text {sat }}\left(T_{\text {ref }}\right)}{p_{\text {sat }}\left(T^{D}\right)}
$$

the heat, which is used for the evaporation process, can be subtracted from the heat of the continuous phase. The temperature $T_{\text {ref }}$ denoting the gas temperature near the drop surface is formulated by the 1/3-law (Hubbard et al. [8]):

$$
T_{\text {ref }}=T^{D}+A_{r}\left(T^{C}-T^{D}\right) \quad \text { with } \quad A_{r}=\frac{1}{3} .
$$

The final two equations completing the Euler/Euler computational scheme are those governing the thermal transport between the phases being represented by the particular set of temperature equations. The liquid mass leaving the droplet in the evaporation process influences strongly both the heat and mass transfer between the phases. This process is accounted for by the Spalding heat and mass transfer 
coefficients $B_{T}$ (Eq. 27) and $B_{M}$ (Eq. 23). This algorithm for their determination is pointed out by the evaporation model proposed by Abramzon and Sirignano [4].

The relative humidity $0 \leq \phi \leq 1$ is defined by the ratio of the vapor mass ratio to its maximum: $\phi=Y / Y_{\text {sat }}$. The mass transfer per surface of a cloud of spherical particles

$$
\begin{aligned}
\frac{\dot{m}}{A_{O}} & =\rho^{C} D_{\alpha \beta} B_{M} \frac{\text { Sh }}{\mathrm{E}\left(D_{p}\right)} \quad \text { with } A_{O}=\pi \mathrm{E}\left(D_{p}^{2}\right) \\
& \Rightarrow \quad \dot{m}=\rho^{C} \pi D_{21} D_{\alpha \beta} \operatorname{Sh} B_{M}
\end{aligned}
$$

depends on the Sherwood Number $S h$ and the mass transfer coefficient $B_{M}$ :

$$
B_{M}=\frac{Y_{\mathrm{sat}}-Y_{\infty}}{1-Y_{\mathrm{sat}}}=\frac{Y_{\mathrm{sat}}}{1-Y_{\mathrm{sat}}}-\frac{Y_{\infty}}{1-Y_{\mathrm{sat}}}
$$

The heat flux

$$
Q_{L}=\mathrm{Nu} \pi D_{21} \lambda^{C}\left(T^{C}-T^{D}\right)-\dot{m} L\left(T^{D}\right)
$$

depending on the Nusselt number $N u$ can also be defined in terms of the heat transfer coefficient $B_{T}$ and the liquid temperature dependent latent heat $L\left(T^{D}\right)$ :

$$
B_{T}=\frac{c_{p}^{D}\left(T^{C}-T^{D}\right)}{Q_{L} / \dot{m}+L\left(T^{D}\right)} \Rightarrow Q_{L}=\dot{m}\left(\frac{c_{p}^{D}\left(T^{C}-T^{D}\right)}{B_{T}}-L\left(T^{D}\right)\right) .
$$

Utilizing the heat flux definition given by Eq. (32), the mass transfer rate formulae can be finally written as a function of the Nusselt number

$$
\dot{m}=\pi D_{21} \frac{\lambda^{C}}{c_{p}^{D}} \mathrm{Nu} B_{T} .
$$

In such a way, the mass transfer from the liquid to the gas phase can be determined modelling the Nusselt number $N u$ and the Spalding Heat transfer coefficient $B_{T}$ with the evaporation model of Abramzon and Sirignano [4].

\section{Computational results and discussion}

The flow configuration simulated of a turbulent, gas/liquid channel flow, being discretized for the quarter channel, is considered. Similar as in the particle-laden cases, a three-dimensional solution domain was adopted. The results obtained by the Euler/Euler computational model were compared with the results obtained by an Euler/Lagrange method (e.g. Sommerfeld et al. [9]) solving the transport equations of the carrier phase (Euler framework) and individual tracking of the droplet parcels (14.800 trajectories were introduced into the flow field displayed in Figs. 2-4.

The boundary conditions of the flow configurations considered (channel dimensions, bulk Reynolds number, turbulence intensities,...) correspond to the 
geometry used for the experimental investigation of the particle-laden flow by Kulick et al. [10]. Instead of solid particles, the flow was laden with droplets characterized by their uniform distribution along the entire inlet cross-section. This flow configuration (no experimental data for the evaporation process exist) with the mass loading of the liquid phase being set to $Z=1 \%$ is simulated, corresponding to a dilute liquid/gas two-phase flow. As already noted, the present evaporation model was assessed by contrasting the Euler/Euler results to the results of the Euler/Lagrange method exclusively.

Figs. 2-4 show some selected results obtained by both approaches. In order to provide the fully-developed flow and turbulence conditions, a completely saturated gas phase (relative humidity was taken to be $100 \%$, outside the left boundary of Fig. 2) was computed over the duct length of $260 h=5.2 \mathrm{~m}$ ( $h$-channel halfwidth), prior to the onset of the evaporation process. The liquid phase evaporation was introduced by heating the duct walls (in such a way the temperature derivative corresponding to the difference between $353 \mathrm{~K}$ prescribed at the inlet cross-section $-x=0$ - and the constant wall temperature of $368 K$ was imposed, Fig. 2) causing a decrease in the relative humidity, Fig. 2. The temperature range $\left(T<100^{\circ} \mathrm{C}\right)$ was chosen to prevent complete evaporation. The work focused primarily on the evaporation process due to concentration gradient. The temperature range prescribed corresponds to the high saturation pressure derivative $d p_{s a t} / d T$ (i.e. $\left.d Y_{\text {sat }} / d T\right)$.

A somewhat slower penetration of the heat flux into the flow core (Fig. 2, lower), obtained by the Euler/Lagrange method in conjunction with original evaporation model of Abramzon and Sirignano [4] implemented in the AVL SWIFT commercial flow solver, causes weaker temperature gradients in this region, leading to a less intensive evaporation process, a fact represented by a slightly higher vapor concentration (weaker decrease from the initial 100\%, Fig. 2 lower). Fig. 3a shows the isolines of the relative Reynolds number (important for the determination of the drag force relaxation time scale $\tau_{p}$ in the volume fraction weighted momentum equation for the continuous phase) based on the
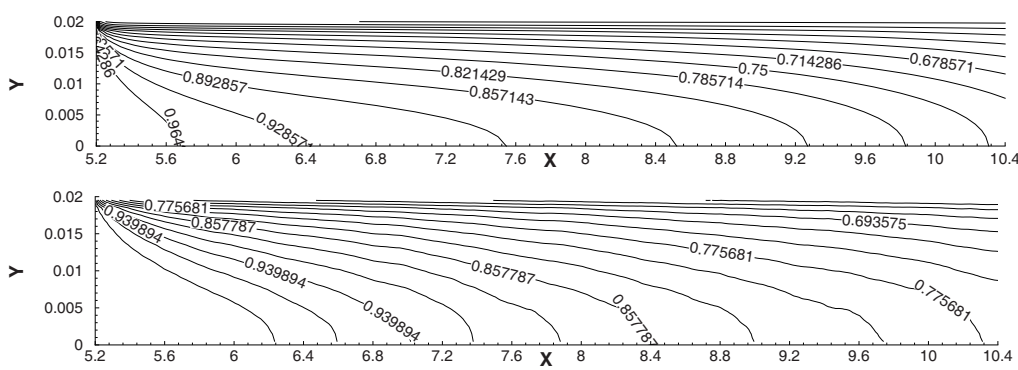

Figure 2: Comparison of the relative humidities obtained by the present Euler/Euler scheme (upper) and the Euler/Lagrange method (lower). 
a)
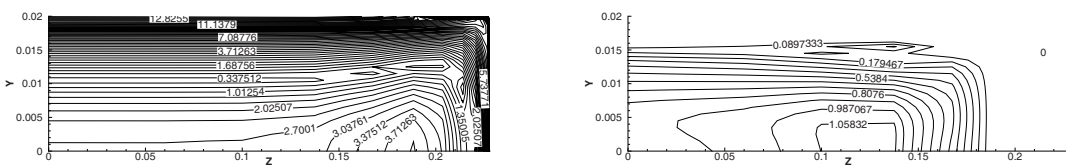

b)
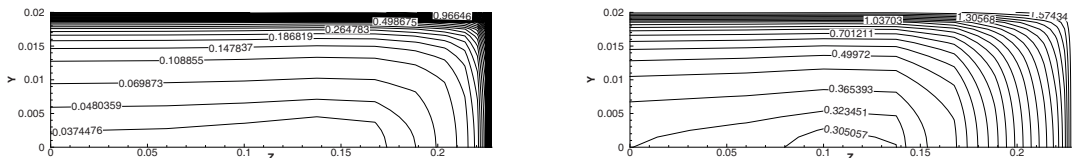

c)
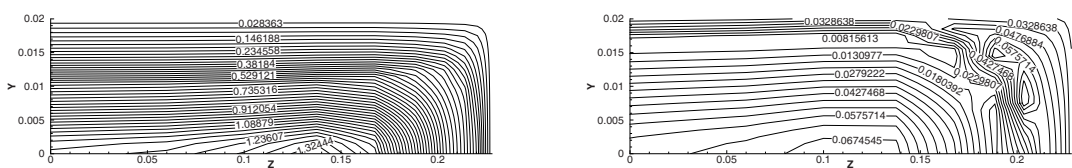

Figure 3: Isolines of a) Reynolds number $R e_{\text {rel }}$, b) mass transfer coefficients $B_{M}$ and c) time scale of evaporation $\tau_{\Gamma}$ across the duct at two selected longitudinal locations $x / h=20$ (left) and 140 (right) obtained by the present Euler/Euler scheme.
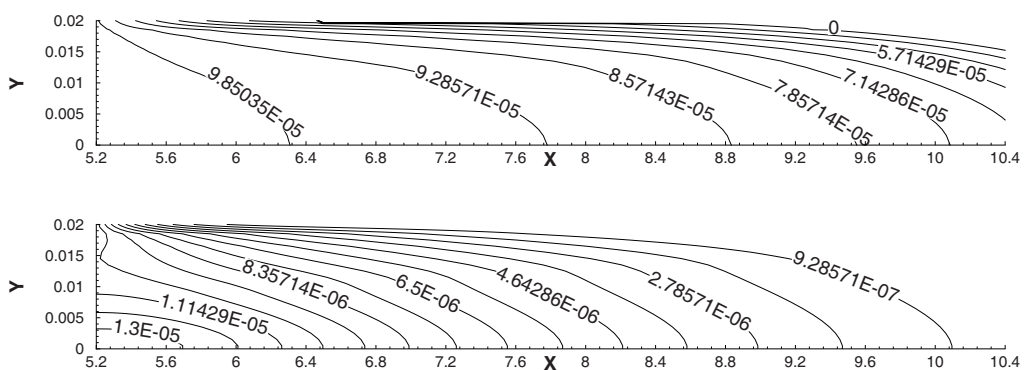

Figure 4: Droplet diameter (upper) and volume fraction $\alpha^{D}$ (lower) isolines obtained by the present Euler/Euler scheme.

velocity difference $\left|\vec{u}^{C}-\vec{u}^{D}\right|$ and droplet diameter $D_{p}$ in the right top quarter of the duct cross-section at two selected longitudinal locations. The results confirm the general reduction of the Reynolds number due to the droplet evaporation (diameter reduction and consequently the volume fraction $\alpha^{D}$ reduction, Fig. 4). This tendency is particularly pronounced in the near-wall flow regions. Fig. 3b displays the evolution of the mass transfer coefficients $B_{M}$ in the Abramzon and Sirignano evaporation model. This coefficient, representing indeed a measure of the vapor fraction being absorbed by the surrounding gas phase, increases due to warm up of the gas phase. The ratio of the droplet surface to the droplet 

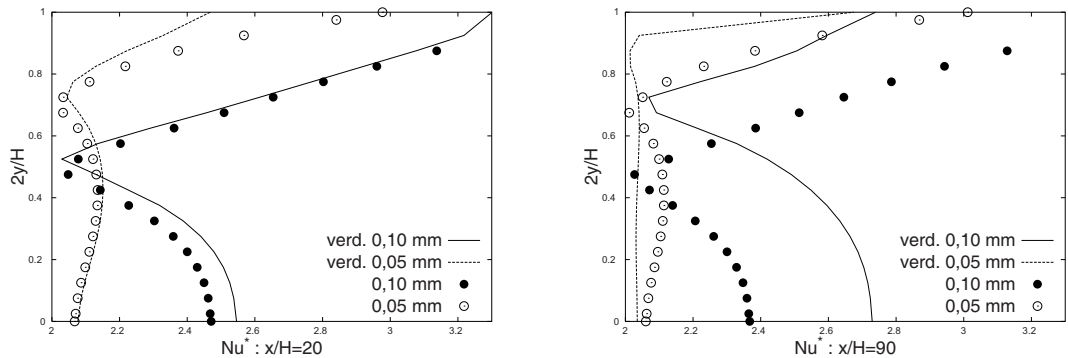

Figure 5: Modified Nusselt-number at two selected locations: $x / h=20$ and 90 .

volume increases by the droplet diameter reduction. Due to the temperature raise, resulting in the intensification of the evaporating process, the time scale of the evaporation is decreasing, Fig. 3c. Fig. 5 illustrates the influence of the evaporation (non-evaporating liquid phase was also computationally simulated) and droplet diameter (two different $D_{p}$-values were analyzed: 50 and $100 \mu \mathrm{m}$ ) on the Nusseltnumber redistribution across the channel. Whereas no significant changes in the Nusselt-number behavior in the case without evaporation (symbols) are noticed, the modified Nusselt number exhibits a decrease in the region with the dominating influence of the small droplets (near wall) if the evaporation is accounted for (lines). In contrast, the increasing effect of the modification (in terms of the Reynolds number, Fig. 3a) is pronounced in the flow core.

\section{References}

[1] Renksizbulut, M. \& Yuen, M.C., Experimental study of droplet evaporation in a high-temperature air stream. ASME Journal of Heat Transfer, 105:384388, 1983.

[2] Park, T.W., Aggarwal, S.K. \& Katta, V.R., Gravity effects on the dynamics of evaporating droplets in a heated jet. Journal of Propulsion and Power, 11(3):519-528, 1995.

[3] Ranz, W.E. \& Marshall W.R., Evaporation from drops: parts I \& II. Chemical Engineering Progress, 48:141-146, 173-180, 1952.

[4] Abramzon, B. \& Sirignano, W.A., Droplet vaporization model for spray combustion calculations. Int. J. Heat Mass Transf., 32:1605-1618, 1989.

[5] Groll, R., Jakirlić, S. \& Tropea, C., Comparative study of Euler/Euler and Euler/Lagrange approaches simulating evaporation in a turbulent gas liquid flow. Int. J. for Num. Meth. in Fluids; 59:873-906, 2009.

[6] Kastner, O., Theoretische und experimentelle Untersuchungen zum Stoffübergang von Einzeltropfen in einem akustischen Rohrlevitator. Dissertation, Technische Fakultät der Universität Erlangen-Nürnberg, 2001.

[7] Politis, S., Prediction of Two-Phase Solid-Liquid Turbulent Flow in Stirred Vessels. PhD Thesis, Imperial College London, 1989. 
[8] Hubbard, G.L., Denny, V.E. \& Mills, S.F., Droplet vaporization - effects on transient and variable properties. Int. J. Heat Mass Transfer, 18:1003-1008, 1975.

[9] Sommerfeld, M., Kohnen, G. Qiu \& H.H., Spray evaporation in turbulent flow: numerical calculations and detailed experiments by phase-doppler anemometry. Revue de Institut Francais du Petrole, 48(6):677-695, 1993.

[10] Kulick, J.D., Fessler, J. \& Eaton, J.K., Particle response and turbulence modification in fully developed channel flow. J. Fluid Mech., 277:109-134, 1994. 